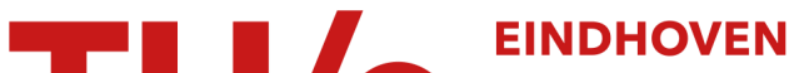 \\ UNIVERSITY OF \\ TECHNOLOGY
}

\section{Diffraction-contrast imaging of cold atoms}

Citation for published version (APA):

Turner, L. D., Domen, K. F. E. M., \& Scholten, R. E. (2005). Diffraction-contrast imaging of cold atoms. Physical Review A : Atomic, Molecular and Optical Physics, 72(3), 031403-1/4. [031403].

https://doi.org/10.1103/PhysRevA.72.031403

DOI:

10.1103/PhysRevA.72.031403

Document status and date:

Published: 01/01/2005

\section{Document Version:}

Publisher's PDF, also known as Version of Record (includes final page, issue and volume numbers)

\section{Please check the document version of this publication:}

- A submitted manuscript is the version of the article upon submission and before peer-review. There can be important differences between the submitted version and the official published version of record. People interested in the research are advised to contact the author for the final version of the publication, or visit the $\mathrm{DOI}$ to the publisher's website.

- The final author version and the galley proof are versions of the publication after peer review.

- The final published version features the final layout of the paper including the volume, issue and page numbers.

Link to publication

\section{General rights}

Copyright and moral rights for the publications made accessible in the public portal are retained by the authors and/or other copyright owners and it is a condition of accessing publications that users recognise and abide by the legal requirements associated with these rights.

- Users may download and print one copy of any publication from the public portal for the purpose of private study or research.

- You may not further distribute the material or use it for any profit-making activity or commercial gain

- You may freely distribute the URL identifying the publication in the public portal.

If the publication is distributed under the terms of Article $25 \mathrm{fa}$ of the Dutch Copyright Act, indicated by the "Taverne" license above, please follow below link for the End User Agreement:

www.tue.nl/taverne

Take down policy

If you believe that this document breaches copyright please contact us at:

openaccess@tue.nl

providing details and we will investigate your claim. 


\title{
Diffraction-contrast imaging of cold atoms
}

\author{
L. D. Turner, ${ }^{*}$, K. F. E. M. Domen, ${ }^{*}$ and R. E. Scholten \\ School of Physics, University of Melbourne, Victoria 3010, Australia
}

(Received 1 March 2005; revised manuscript received 17 May 2005; published 15 September 2005)

\begin{abstract}
We consider the inverse problem of in-line holography, applied to minimally destructive imaging of cold atom clouds. Absorption imaging near resonance provides a simple, but destructive measurement of atom column density. Imaging off resonance greatly reduces heating, and sequential images may be taken. Under the conditions required for off-resonant imaging, the generally intractable inverse problem may be linearized. A minimally destructive, quantitative and high-resolution image of the atom cloud column density is then retrieved from a single diffraction pattern.
\end{abstract}

DOI: 10.1103/PhysRevA.72.031403

PACS number(s): 32.80.Pj, 42.40.Ht, 42.30.Rx

The simplest optical measurement of object structure is made by illuminating the object with radiation and recording the diffraction pattern produced (Fig. 1). The inverse problem of retrieving the structure of a noncrystalline object from its Fresnel diffraction pattern has been studied since Gabor's first incomplete solution, known as in-line holography [1]. This Communication solves the particular inverse problem of retrieving the structure of a cold atom cloud from a single diffraction pattern.

Minimally destructive imaging of cold atoms requires weak absorption. High-resolution imaging also places constraints on phase shifts. Under these assumptions, we derive a linear solution which retrieves the column density of the atom cloud from a single diffraction pattern. We apply this solution to demonstrate off-resonant imaging of a cold atom cloud without beamsplitters, phase plates, or imaging optics.

Gabor's in-line holography recovers an approximation of the original wavefield by illuminating a photographic transparency of the diffraction pattern. The reconstructed wavefield is contaminated by the superimposition of an out-offocus twin image [2]. Other forms of holography use a reference beam to record an interference pattern, rather than a diffraction pattern, and so separate the twin image [3]. In this Communication we demonstrate a nonholographic method of retrieval. Such methods have been proposed when it is inconvenient or impossible to generate a coherent reference beam. The first step is common with Gabor's method: a diffraction pattern is recorded without the need for optics such as lenses and beamsplitters. In the second step, rather than reconstructing the wavefield by optical propagation (physical or numerical), an image is extracted by solving an inverse problem with specified constraints.

There is insufficient information in a single intensity image to retrieve both the amplitude and the phase of the wavefield. This information deficit may be balanced, and the inverse problem solved, if the object is assumed to be purely

\footnotetext{
*Present address: National Institute of Standards and Technology, Gaithersburg, Maryland 20899, USA

†Electronic address: 1.turner@aip.org.au

${ }^{*}$ Present address: Faculty of Applied Physics, Eindhoven University of Technology, The Netherlands.
}

absorbing [4] or purely phase shifting [5], but these assumptions are seldom valid in practice.

Instead, we present a single-image solution based on the assumption of a monomorphous object (one made of a single material), so that both the phase shift $\phi$ and the absorption $\mu$ of the object are proportional to the column density of material along the optical path $\rho(\mathbf{x})=\int_{-\infty}^{0} N(\mathbf{r}) \mathrm{d} z$ :

$$
\mu(\mathbf{x})=k \beta \rho(\mathbf{x}) \quad \text { and } \quad \phi(\mathbf{x})=k \delta \rho(\mathbf{x}) .
$$

The variable $\mathbf{x}$ represents coordinates in a plane transverse to the incident wave propagating along the $z$ axis and $k$ $=2 \pi / \lambda$ is the wave number for illuminating radiation of wavelength $\lambda$. The absorption and phase coefficients $\beta$ and $\delta$ correspond to a refractive index of the form

$$
n=1+N(\mathbf{r})(\delta+i \beta)
$$

with $N(\mathbf{r})$ the atom number density. This monomorphous object assumption has been used in compensating defocus and spherical aberration in electron micrographs [6] and transport-of-intensity imaging [7].

Immediately after an optically thin object [Fig. 1(a)], an incident scalar plane wave of amplitude $f_{0}$ becomes $f(\mathbf{x})$ $=f_{0} \exp (-\mu(\mathbf{x})+i \phi(\mathbf{x}))$, and this wavefield may be propagated through a distance $z$ by the Fresnel transform

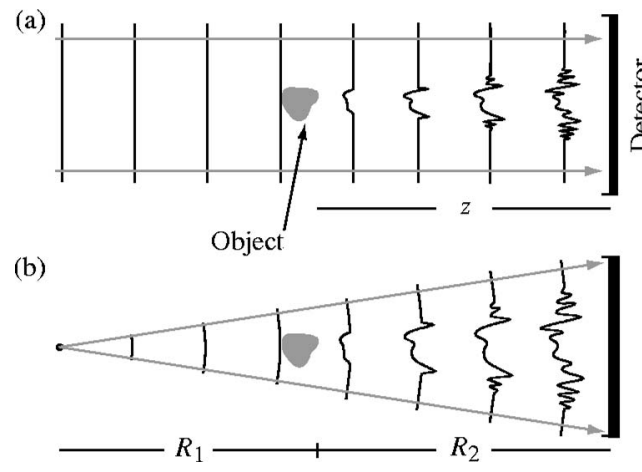

FIG. 1. Recording a diffraction pattern. Vertical lines represent intensity profiles. In part (b), point-source illumination magnifies the diffraction pattern. 


$$
f(\mathbf{x}, z)=\frac{\exp (i k z)}{i \lambda z} \int_{-\infty}^{+\infty} f(\mathbf{x}) \exp \left(\frac{i \pi}{\lambda z}\left|\mathbf{x}-\mathbf{x}^{\prime}\right|^{2}\right) \mathrm{d} \mathbf{x}^{\prime} .
$$

The Fresnel approximation agrees closely with the complete scalar diffraction theory, except for propagation at large angles to the axis or within a few wavelengths of the object. Optical detectors measure intensity $I=|f|^{2}$ and it can be shown [8] that the Fourier transform $\mathcal{F}$ of the diffracted intensity measured at $z$ can be expressed in terms of the objectplane wavefield $f(\mathbf{x})$ as

$$
\begin{aligned}
\mathcal{F}[I(\mathbf{x}, z)]= & \int_{-\infty}^{+\infty} f^{*}(\mathbf{x}+\lambda z \mathbf{u} / 2) f(\mathbf{x}-\lambda z \mathbf{u} / 2) \\
& \times \exp (-2 \pi i \mathbf{x} \cdot \mathbf{u}) \mathrm{d} \mathbf{x},
\end{aligned}
$$

in which $\mathbf{u}$ is the spatial frequency conjugate to $\mathbf{x}$. Written in terms of absorption and phase shifts, this is

$$
\begin{aligned}
\mathcal{F}[I(\mathbf{x}, z)]= & I_{0} \int_{-\infty}^{+\infty} \exp \{-\mu(\mathbf{x}+\lambda z \mathbf{u} / 2)-\mu(\mathbf{x}-\lambda z \mathbf{u} / 2) \\
& +i[\phi(\mathbf{x}-\lambda z \mathbf{u} / 2)-\phi(\mathbf{x}+\lambda z \mathbf{u} / 2)]\} \\
& \times \exp (-2 \pi i \mathbf{x} \cdot \mathbf{u}) \mathrm{d} \mathbf{x} .
\end{aligned}
$$

Assuming both real and imaginary parts of the exponential are small, we expand and apply the Fourier shift theorem to yield

$$
\begin{aligned}
\mathcal{F}[I(\mathbf{x}, z)]= & I_{0}\left(\delta(\mathbf{u})-2 \cos \left(\pi \lambda z u^{2}\right) \mathcal{F}[\mu(\mathbf{x})]\right. \\
& \left.+2 \sin \left(\pi \lambda z u^{2}\right) \mathcal{F}[\phi(\mathbf{x})]\right) .
\end{aligned}
$$

This expression $[10,9]$ relates absorption and phase shift to the intensity of the diffraction pattern. The linearizing assumption used in obtaining Eq. (6) implies

$$
\begin{gathered}
2 \mu(\mathbf{x}) \ll 1 \\
\text { and }|\phi(\mathbf{x}+\lambda z \mathbf{u} / 2)-\phi(\mathbf{x}-\lambda z \mathbf{u} / 2)| \ll 1 .
\end{gathered}
$$

The object must not be strongly absorbing, but it need not be completely transparent. The phase shift should obey the finite difference condition Eq. (8), which restricts large variations in the phase shift to coarse structures in the object. Note that weak phase shift $(|\phi(\mathbf{x})| \ll 1)$ is sufficient to satisfy Eq. (8) but is not necessary [9]. This phase condition may always be met at small $z$, but phase objects of many radians thickness may require impractically small propagation distances, and phase shifts of order 1 radian are preferable.

For monomorphous objects obeying Eq. (2), there is then a linear shift-invariant relation between the normalized contrast $I / I_{0}-1$ and the column density $\rho$ :

$$
\mathcal{F}\left[\frac{I-I_{0}}{I_{0}}\right]=2 k\left(\delta \sin \left(\pi \lambda z u^{2}\right)-\beta \cos \left(\pi \lambda z u^{2}\right)\right) \mathcal{F}[\rho(\mathbf{x})] .
$$

The factor $\widetilde{h}(u ; z)=\delta \sin \left(\pi \lambda z u^{2}\right)-\beta \cos \left(\pi \lambda z u^{2}\right)$ is termed the contrast transfer function (CTF), and is plotted in Fig. 2 for positive and negative values of the phase coefficient $\delta$. Equation (9) can be solved formally for $\rho$, but the zeros in the

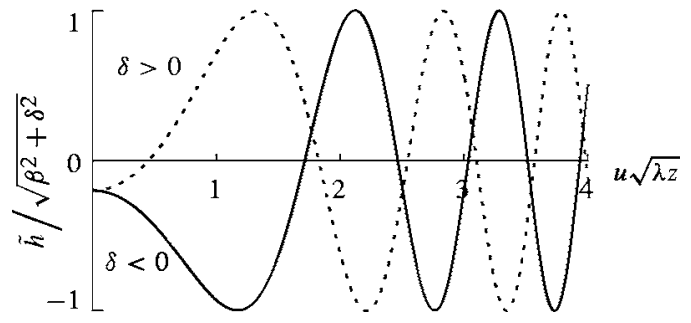

FIG. 2. The contrast transfer function $\tilde{h}$, normalized, for phaseadvancing (solid line) and phase-retarding (dashed line) monomorphous objects. At low spatial frequencies the contrast approaches the value for an in-focus absorption image.

CTF render the retrieval an ill-posed inverse problem.

The inverse problem may be regularized, for example by the Tikhonov method [11]. Rather than dividing $\mathcal{F}\left[I / I_{0}-1\right]$ by $\tilde{h}(u ; z)$, the Tikhonov filter retrieves the column density by the modified division

$$
\rho(\mathbf{x})=\frac{1}{2 k} \mathcal{F}^{-1}\left[\frac{\tilde{h}(u ; z)}{\widetilde{h}^{2}(u ; z)+\alpha^{2}} \mathcal{F}\left[\frac{I-I_{0}}{I_{0}}\right]\right]
$$

which closely approximates division by the CTF except at spatial frequencies where the CTF is near zero. Larger values of the Tikhonov parameter $\alpha$ reduce the amplification of noise in the retrieval process, but at the expense of image distortions. Smaller values yield less distorted but noisier retrievals; a normalized alpha value of 0.2 was used. Algorithmic optimization of $\alpha$ is possible, for example using Fourier-wavelet regularized deconvolution [12].

It is clear from Fig. 2 that the solution is more stable if the object advances the phase of the incident wave $(\delta<0)$, and the CTF zero crossing at low spatial frequencies is avoided. The column density may be retrieved for phase-retarding objects $(\delta>0)$ but, as shown in Fig. 2, the focusing action of the phase shift cancels the absorption contrast at low spatial frequencies and lower quality retrievals result.

If the diffraction pattern is re-imaged by a lens, the system may be defocused behind the object so that the effective propagation distance $z$ is negative. It follows from Eq. (9) that the sign condition on $\delta$ is then reversed. For lensless imaging, negative $z$ cannot be achieved and the object should be phase advancing.

Magnified images can be retrieved even without lenses. Rather than using plane-wave illumination, a point source of light a distance $R_{1}$ before the object produces a spherical wave incident on the object [Fig. 1(b)]. The diffraction at detector distance $R_{2}$ is magnified by the geometric factor $M=\left(R_{1}+R_{2}\right) / R_{1}$, but is otherwise identical to the plane-wave pattern of Fig. 1(a) at the effective propagation distance $z_{\text {eff }}$ $=R_{2} / M[13]$.

Conventional optical materials are phase retarding $(\delta$ $>0$ ) but for x-ray imaging [9], and for imaging atomic gases with light blue detuned from an atomic resonance, the phase is advanced. We now show that our solution to the diffraction imaging inverse problem is exactly suited to off-resonant imaging of cold atom clouds.

To date, all measurements of ground-state BEC's have 
been made with near-resonant optical probes. On-resonance absorption imaging is destructive for most BEC configurations. Imaging with an off-resonant probe reduces heating due to spontaneous emission, with the cloud instead shifting the phase of the probe beam. Dark-field [14] and Zernike phase-contrast [15] techniques of phase microscopy have been used to render these phase-shifts visible and hence obtain sequential, minimally destructive images of BEC. Other methods investigated for off-resonant imaging include an interferometric technique equivalent to off-axis image holography [16] and a propagation-contrast method based on transport of intensity [17]. Although minimally destructive imaging has been crucial to observing many dynamic processes in BEC, destructive absorption imaging is still widely used.

As shown above, free space propagation produces phase contrast without optics. Gaussian fits to atom cloud images (peak column density and diameters only) have been extracted from fitting diffraction patterns [18]. Our solution to the inverse problem retrieves detailed column-density images of cold atom clouds, without requiring Zernike phase plates or interferometry.

In the two-level approximation, the refractive index of an atomic gas is

$$
n=1+N(\mathbf{r}) \frac{\sigma_{0} \lambda}{4 \pi} \frac{i-2 \Delta}{1+4 \Delta^{2}},
$$

where $N$ is the number density of atoms, $\Delta$ is the detuning in natural linewidths, and $\sigma_{0}$ is the resonant cross section $\left(3 \lambda^{2} / 2 \pi\right.$ for closed transitions). Comparison with Eq. (2) confirms that such an atomic cloud is a monomorphous object, with absorption and phase shifts through the cloud given by Eq. (1). Provided that the atom cloud satisfies the weak-absorption condition Eq. (7) and limited-phase condition Eq. (8), the CTF relation Eq. (9) applies. Substituting the $\beta$ and $\delta$ coefficients from Eq. (11) yields the cold atom $\mathrm{CTF}$

$$
\tilde{h}(u ; z)=-\frac{\sigma_{0}}{2 k\left(1+4 \Delta^{2}\right)}\left[2 \Delta \sin \left(\pi \lambda z u^{2}\right)+\cos \left(\pi \lambda z u^{2}\right)\right],
$$

which is then used in Eq. (10) to retrieve the column density of the atom cloud. If the detuning is blue of resonance, the atom cloud advances the phase of the incident light, and the low-frequency CTF zero is avoided.

An important feature of this application to cold atom imaging is the regularizing effect of residual absorption. At zero spatial frequency, the CTF falls to $\sim 1 / 2 \Delta$ of its maximum value, but does not vanish completely as it would for a pure phase object. Even small residual absorption is sufficient to stabilize the retrieval and then the Tikhonov modified form Eq. (10) need only be used at higher spatial frequencies above $u_{\min }=1 / \sqrt{2 \lambda z}$. Such partial regularization greatly reduces distortion, and retrievals approach the optimal linear estimate (Wiener filter) which can only be calculated with full advance knowledge of the object power spectrum.

This linearization of the inverse problem is only valid if the atom cloud meets the absorption and phase conditions
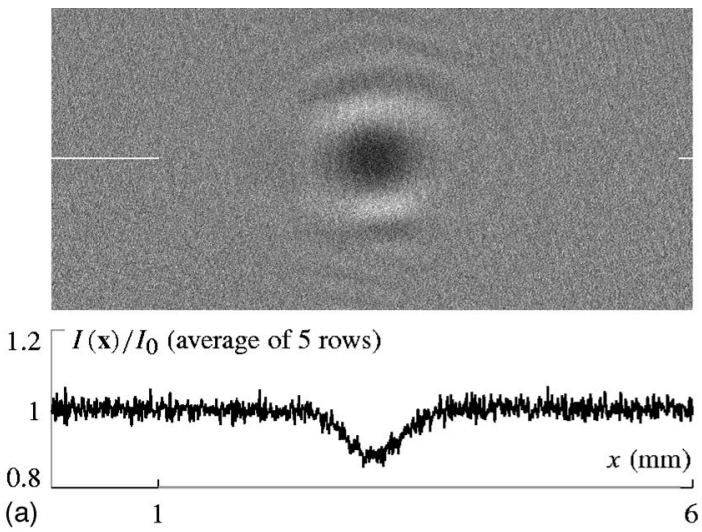

(b)
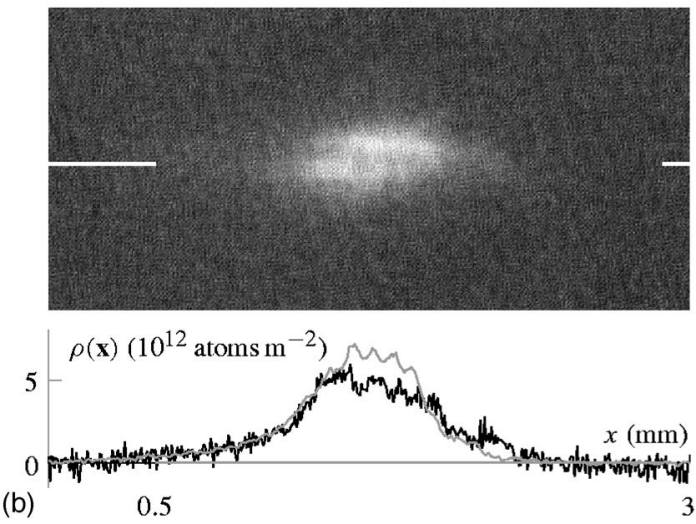

FIG. 3. Above, the recorded diffraction pattern. Below, the column density retrieved using Eq. (10), shown at twice the magnification of the diffraction pattern. Plots averaged over the central five rows of pixels are shown below the images. The overplotted gray trace shows the column density calculated from an on-resonance in-focus absorption image.

[(7) and (8)]. Minimally destructive imaging necessarily obeys the weak-absorption condition. The phase condition Eq. (8) also broadly concurs with physical constraints due to refraction and resolution. Light refracted by the object must remain within the numerical aperture of the lens (or detector) [14]. It follows that structures at the diffraction limit of the imaging system should have phase variations less than one radian. Objects satisfying this "thin object" condition-that the detailed structure of the object must vary by less than a radian-are likely to also satisfy the slowly varying phase condition Eq. (8).

Detuning the probe light by $\rho_{\max } \sigma_{0} / 4$ full linewidths from resonance reduces the peak phase-shift to order one radian. BEC's typically have resonant optical densities $\rho_{\max } \sigma_{0}$ $\approx 300$ and so detunings must be of order $100 \Gamma$ to meet the refraction condition. At such detunings, many images may be taken before the cloud is appreciably heated. In the shotnoise limit, further increasing detuning and intensity does not improve the SNR beyond a limiting value, and in the presence of technical noise will reduce the SNR. It has been shown that this SNR limit is determined only by the number of spontaneous emission events and condensate parameters [19].

In proof-of-principle experiments, the point-projection configuration of Fig. 1(b) was used to image a cold atom 
cloud of peak resonant optical density 2.2. A weak linearly polarized probe beam, detuned $+3.1 \Gamma$ from the ${ }^{85} \mathrm{Rb}_{4} \mathrm{~S}_{1 / 2}(F=3) \rightarrow 5 \mathrm{P}_{3 / 2}(F=4)$ transition, diverged from the cleaved endface of a single-mode optical fiber, expanding for $R_{1}=125 \mathrm{~mm}$ before passing though a cloud of cold atoms around $300 \mu \mathrm{m}$ in diameter held in a magneto-optical trap (MOT) vacuum chamber. Trapping beams were turned off for the $300 \mu$ s duration of the probe pulse. The beam propagated a further distance $R_{2}=155 \mathrm{~mm}$ to a CCD camera (Photometrics Coolsnap HQ, $1392 \times 1040$ pixels, $6.45 \mu \mathrm{m}$ pitch), producing the diffraction pattern shown in Fig. 3. The column-density image was retrieved using Eq. (10); retrievals take around one second on a Pentium-III processor using standard discrete Fourier transform algorithms [20].

It is usually not possible to measure the propagation distances $R_{1}$ and $R_{2}$ accurately enough to produce an optimal retrieval. Instead, the retrieval is performed with the contrast transfer function $\widetilde{h}(u ; z)$ evaluated at various values of $z$ until a sharp image is retrieved. Thus focusing is performed in software when retrieving, rather than by adjusting lens positions when imaging. As a corollary, the retrievals show holographic depth-of-field: one diffraction pattern can be used to retrieve images at many different $z$ values. The very real problems of focusing the optics, and of the limited depth-offield inherent in high-resolution imaging, are completely obviated.

While the precise setting and knowledge of the propagation distance is immaterial, its coarse setting affects the shape and contrast of the diffraction pattern and hence the signal-to-noise ratio (SNR) of the retrieved image. At short distances only residual absorption contrast will be rendered. At large propagation distances and for small phase-shifts, the root-mean-square SNR approaches $71 \%$ of that obtained with the Zernike technique [21].

A further advantage of the point-projection configuration is the absence of lenses, and their resolution-limiting aberrations. Diffraction contrast may also be produced by defocusing an existing absorption imaging system, which may be more convenient than placing the camera very close to the object. The advantages of holographic depth-of-field and post-hoc focusing are retained.

In practice, the propagation distance is constrained by resolution limits. For a detector of diameter $D$, the minimum resolvable line spacing in the retrieved image is of order $2 \lambda R_{2} / D$, as it is for a lens of the same diameter in the same position. The $R_{1}$ distance should then be chosen to provide sufficient magnification that resolution is limited by diffraction and not by the pixel size. In our experiment, optical access limited the resolution to $30 \mu \mathrm{m}$. We can predict that a $\mathrm{BEC}$ in a glass cell imaged with $R_{1}=12 \mathrm{~mm}$ and $R_{2}$ $=60 \mathrm{~mm}$ on a $D=25 \mathrm{~mm} C C D$ with $9 \mu \mathrm{m}$ pixels yields a pixel resolution of $3 \mu \mathrm{m}$ and a diffraction-limited resolution of $3.7 \mu \mathrm{m}$. Further, the CTF depends on $z, \lambda, \Delta$ and $u$. Once $z$ is set by "focusing," the remaining quantities are readily measured to better than $1 \%$. Such well-defined parameters and the lack of lens aberrations yield highly quantitative column density measurements.

We solve the inverse problem of retrieving a quantitative column density image from a single diffraction pattern by exploiting the proportionality between absorption and phase shift through a single-material object. The predicates of the solution are uniquely suited to imaging cold atom clouds. Lens aberrations are precluded by avoiding the need for image formation entirely. Beam-splitters, phase-plates, and other optical elements are also unnecessary. The holographic record allows refocusing after the image has been acquired. The solution can be used with existing absorption imaging systems simply by defocusing the imaging lens. We calculate near-wavelength resolution when using point-projection to image Bose-Einstein condensates.
[1] D. Gabor, Nature (London) 161, 777 (1948).

[2] J. W. Goodman, Introduction to Fourier Optics (McGraw-Hill, Boston, 1996), p. 303.

[3] E. N. Leith and J. Upatnieks, J. Opt. Soc. Am. 54, 1295 (1964).

[4] L. Onural and P. D. Scott, Opt. Eng. 26, 1124 (1987).

[5] P. Cloetens et al., Appl. Phys. Lett. 75, 2912 (1999).

[6] H. P. Erickson and A. Klug, Philos. Trans. R. Soc. London, Ser. B 261, 105 (1971).

[7] D. Paganin et al., J. Microsc. 206, 33 (2002).

[8] J.-P. Guigay, Optik (Stuttgart) 49, 121 (1977).

[9] L. D. Turner et al., Opt. Express 12, 2960 (2004).

[10] E. Menzel, Optik (Stuttgart) 15, 460 (1958).

[11] A. N. Tikhonov and V. Y. Arsenin, Solutions of Ill-posed Problems (V. H. Winston, Washington, D.C., 1977).

[12] R. Neelamani, Hyeokho Choi, and R. Baraniuk, IEEE Trans. Signal Process. 52, 418.
[13] A. Pogany, D. Gao, and S. W. Wilkins, Rev. Sci. Instrum. 68, 2774 (1997).

[14] M. R. Andrews et al., Science 273, 84 (1996).

[15] M. R. Andrews et al., Phys. Rev. Lett. 79, 553 (1997).

[16] S. Kadlecek, J. Sebby, R. Newell, and T. G. Walker, Opt. Lett. 26, 137 (2001).

[17] L. D. Turner, K. P. Weber, D. Paganin, and R. E. Scholten, Opt. Lett. 29, 232 (2004).

[18] D. Meschede et al., Opt. Commun. 145, 57 (1998).

[19] J. E. Lye, J. J. Hope, and J. D. Close, Phys. Rev. A 67, 043609 (2003); J. J. Hope and J. D. Close, Phys. Rev. Lett. 93, 180402 (2004).

[20] Diffraction-contrast imaging (software). http://eprints.unimelb.edu.au/archive/835

[21] L. D. Turner, Ph.D. thesis, University of Melbourne, 2004. http://eprints.unimelb.edu.au/archive/822 